Editorial

\title{
Bacteriophages offer an antimicrobial solution
}

\section{Introduction}

The antimicrobial crisis has been reported in depth. ${ }^{1}$ Modern society faces the risk of a future without effective antibiotics and antimicrobials, against a number of pathogenic strains. This is due to the rise in antimicrobial resistance, which is the result of changes in microbial genes (spontaneous or induced genetic mutations). Acquired resistance has developed with several pathogenic bacteria due to the over-use of antimicrobials (such as mis-prescribing and the use of antibiotics on farm animals in an attempt to rear leaner meat). ${ }^{2}$ The future consequences of this are a reduction in life expectancy due to people dying from diseases that are readily treatable today and the lower the chances of recovering from different surgical procedures. ${ }^{3}$

The search for new antimicrobials, although given renewed focus in the past decade, is proving difficult, although there are some interesting candidate compounds emerging. ${ }^{4}$ A key reason for the slow-rate of progress has been the limited funding for research and development, initiated by the pharmaceutical sector due to the low return-on-investment. This has led to governments and the university sector spearheading much of the recent research.

One potential area for antimicrobial therapy, which takes a different direction to the search for new compounds, is with bacteriophages: viruses that have the ability to infect and fight harmful bacteria.

\section{Bacteriophages}

Bacteriophages have been known of for over one hundred years. They were first described by the British bacteriologist Twort $^{5}$ in 1915 and the French-Canadian scientist Felix d'Herelle in 1917. Bacteriophages are viruses capable of infecting a bacterium and replicating within the cell (through injection of the viral genome into the bacterial cell cytoplasm). While bacteriophages vary in their complexity, they are made-up of proteins which encapsulate either a DNA or RNA genome. ${ }^{6,7}$ Orders include Caudovirales and Ligamenvirales. Bacteriophages are found in water, soil and mammalian intestines, in high numbers. In seawater, for example, there are some $9 \times 10^{8}$ virions per millilitre of water. ${ }^{8}$

\section{Phage therapy}

As part of the antimicrobial battle, different research teams have been examining the use of bacteriophages as a potential therapy to combat multi-drug-resistant bacterial pathogens. This is not a new concept, for d'Herelle himself proposed the idea in 1919 and undertook initial research. Following this, phages have been used as antibiotic alternatives in some countries, particularly those in Eastern Europe, since the 1930s. However, the discovery of various antibiotics in subsequent decades, as well as problems experienced with the use of phages, forestalled research in phage therapy. Consequently, clinical therapies have not, until recently, advanced significantly.

The advantage of phage therapy is that the bacteriophage is easy to administer and, on entering the host bacterium, the virus will replicate readily. Where biofilms are present, phages have been shown to be more effective at penetrating the bacterial cell wall than antimicrobial compounds. ${ }^{9}$ One entering the bacterial cell, phages release lysins that target enzymes in the bacterial cell wall.
Volume 3 Issue I - 2016

\author{
Tim Sandle \\ Pharmig (Pharmaceutical Microbiology Interest Group), \\ Stanstead Abbotts, UK
}

Correspondence: Tim Sandle, Pharmig (Pharmaceutical Microbiology Interest Group), Stanstead Abbotts, UK, Email timsandle@btinternet.com

Received: January 06, 2016 | Published: January 07, 2016

A downside is that phages are host-specific and the correct phage needs to be used against a specific pathogen or serotype. This could limit some types of phage therapy to the emerging field of personalised medicines, rather than providing broad spectrum antimicrobial solutions. Furthermore, bacteria can become resistant to phages although this is unlikely to be to the same extent as the acquired resistance by bacteria to antimicrobials. Moreover, phages have proven to be redify modifiable in laboratory tests.

A further difficulty with the use of phages is the body's own defence system, where phages are often rapidly eliminated by the reticuloendothelial system.

To enable the selection of the appropriate phage for different bacteria, more advanced and rapid microbiological methods are required. Some relatively new techniques have helped to make the selection easier. Advances in such diagnostic methods as realtime PCR, 16s rRNA sequencing and laser-induced breakdown spectroscopy, as examples, have enabled the more accurate assessment of phages with potential therapeutic application. ${ }^{10}$

Using such diagnostic methods, advances are being made. In relation to food safety, for example, United States Department of Agriculture scientists have identified three Escherichia coli O157:H7-specific bacteriophages (ECP-100). In trials, the phages proved successful in reducing the population of $E$. coli $\mathrm{O} 157: \mathrm{H} 7$ on contaminated lettuce. ${ }^{11}$ In medicine, successful outcomes were obtained using phage therapy in curing chronic ear infections caused by Pseudomonas aeruginosa, ${ }^{12}$ as well as with treating urogenital tract infections and sepsis, ${ }^{13}$ and a case of ling disease caused by Burkholderia cenocepacia, where intraperitoneal administration of a phage therapy was found to be superior to the intranasal route. ${ }^{14}$

A further advantage with phage therapy, when used as a type of biological 'smart bomb', is that the process theoretically elicits very few side-effects for the patient and phages seem innocuous. ${ }^{15}$

\section{Summary}

Phage therapy will not provide a complete antimicrobial solution and research into alternative therapeutic compounds to add to the array of antimicrobials available remains an important research area. Nonetheless, phage therapy is an area with great potential and its wider use would complement the use of antimicrobial drugs. 
Phage therapy can provide an effective means for combating multidrug resistant bacteria. In theory, it is straightforward to administer and carries a low risk for the patient. However, to be fully realized further research is required and one obstacle to hampering this is an ill-defined and incomplete regulatory structure. Reform of research guidelines and stipulations for clinical trials, from bodies like the U.S. Food and Drug Administration, would help to push research into novel phage therapy forwards.

\section{Acknowledgments}

None.

\section{Conflicts of interest}

Author declares that there is no conflict of interest.

\section{Reference}

1. Sandle T. Novel methods to address antimicrobial resistance. SOJ Microbiol Infect Dis. 2014;2(1):2-3.

2. Sandle T. Searching for Antimicrobials in the unlikeliest of places. $J$ Pharm Microbiol. 2015;1(1):1-3.

3. Barnett ML, Linder JA. Antibiotic prescribing to adults with sore throat in the United States 1997-2010. JAMA Intern Med. 2014;174(1):138-140.

4. Sandle T. Teixobactin: a new class of antibiotic. SOJ Microbiol Infect Dis. 2015;3(1):1-2.

5. Twort FW. The Discovery of the "bacteriophage". The Lancet $1925 ; 205(5303): 845$.
6. Keen EC. Felix d'Herelle and our microbial future. Future Microbiol. 2012;7(12):1337-1339.

7. Deresinski S. Bacteriophage therapy: exploiting smaller fleas. Clin Infect Dis. 2009;48(8):1096-1101.

8. Wommack KE, Colwell RR. Virioplankton: viruses in aquatic ecosystems. Microbiol Mol Biol Rev. 2000;64(1):69-114.

9. Pirisi A. Phage therapy-advantages over antibiotics? Lancet. 2000;356(9239):1418.

10. Rhoads D, Wolcott R, Sun Y, et al. Comparison of culture and molecular identification of bacteria in chronic wounds. Int $\mathrm{J} \mathrm{Mol} \mathrm{Sci}$. 2012;13(13):2535-2550.

11. Sharma M, Patel JR, Conway WS, et al. Effectiveness of bacteriophages in reducing Escherichia coli $\mathrm{O} 157: \mathrm{H} 7$ on fresh-cut cantaloupes and lettuce. J Food Prot. 2009;72(7):1481-1485.

12. Wright A, Hawkins CH, Anggard EE, et al. A controlled clinical trial of a therapeutic bacteriophage preparation in chronic otitis due to antibioticresistant Pseudomonas aeruginosa: a peliminary report of efficacy. Clin Otolaryngol. 2009;34(4):349-357.

13. Abedon ST, Kuhl SJ, Blasdel BG, et al. Phage treatment of human infections. Bacteriophage. 2011;1(2):66-85.

14. Carmody LA, Gill JJ, Summer EJ, et al. Efficacy of bacteriophage therapy in a model of Burkholderia cenocepacia pulmonary infection. $J$ Infect Dis. 2010;201(2):264-271.

15. Rhoads DD, Wolcott RD, Kuskowski MA, et al. Bacteriophage therapy of various leg ulcers in humans: results of a phase I safety trial. $J$ Wound Care. 2009;18(6):237-243. 